\title{
Developing a materials world: an analysis of UK higher education data
}

\author{
Eleonora D'Elia ${ }^{a}$, Alessandro Mottura ${ }^{b, *}$, Ben Britton ${ }^{d, a}$, Han Zhang ${ }^{c}$, Christopher \\ Hamlett ${ }^{\mathrm{b}, \mathrm{e}}$ \\ ${ }^{a}$ Department of Materials, Imperial College London \\ ${ }^{b}$ School of Metallurgy and Materials, University of Birmingham \\ 'School of Engineering and Materials Science, Queen Mary University of London \\ ${ }^{d}$ Department of Materials, University of British Columbia \\ e Henry Royce Institute
}

\begin{abstract}
Despite being a catalyst for progress, Materials Science and Engineering is relatively unknown as an undergraduate discipline in the UK. By analysing UK data published by the Universities and Colleges Admissions System (UCAS) and the United Kingdom's Higher Education Statistics Agency (HESA), we explore how applications to study and uptake of Materials Science and Engineering differs from other related disciplines in the United Kingdom. We find Materials Science and Engineering lags significantly behind related disciplines in terms of applications, and this is likely to have ramifications on the diversity of our student population. We also find a significant number of students is likely to join a Materials Science and Engineering programme by an internal transfer process rather than by direct application. Our analysis suggests higher education institutions should ensure Materials Science and Engineering is defined well as a discipline, both in marketing materials and by consistently using the Higher Education Classification of Subjects (HECoS) coding system.
\end{abstract}

Keywords: Materials Science and Engineering, Higher Education, United Kingdom

\footnotetext{
* Corresponding author

Email address: a.mottura@bham.ac.uk (Alessandro Mottura)
} 


\section{Contents}

\begin{tabular}{lll}
\hline & Introduction & 2
\end{tabular}

2 Applying to University in the United Kingdom 3

3 Classification of subjects

4 UCAS and HESA data $\quad 6$

\begin{tabular}{|lll}
5 & Coding of Materials Science and Engineering & 7
\end{tabular}

6 Applications to study Materials Science and Engineering 9

$\begin{array}{lll}7 & \text { Characteristics of applicants pool } & 13\end{array}$

7.1 Women . . . . . . . . . . . . . . . . . . . . . . 13

7.2 Ethnic background . . . . . . . . . . . . . . . . . . . . 14

7.3 Socioeconomic background . . . . . . . . . . . . . . . . . 15

\begin{tabular}{lll}
\hline 8 & Conversion to students & 17
\end{tabular}

\section{Introduction}

This article is intended to accompany Developing a materials world: why (not) us?, an article published in the IOM3 magazine, Materials World (March 2021).

Due to space limitation in the published article, a full explanation of how the data was analysed could not be included, and some data could not be presented. Also, some readers may benefit from a detailed description of how the Universities and Colleges Admissions System (UCAS) and the United Kingdom's Higher Education Statistics Agency (HESA) data is collected and published. We hope this may help UK Materials Departments to make informed choices when deciding how their data is reported to HESA, as well as assist them in developing equality, diversity and inclusion strategies.

12 In this article we will first briefly describe the UK University application process, 13 for those readers that are unfamiliar with it (Section 2). We will then explain how 14 disciplines are classified by nationwide bodies (Section 3) and how data on applica-

15 tions and students is collected and published (Section 4). We will then introduce 
how MSE is represented (and affected) by the subject coding systems (Section 5). Finally, we compare the overall number of home and overseas applications to MSE courses with other STEM subjects (Section 6), the personal characteristics of the applicants pool (Section 7) and then analyse the conversion from applicants to students in Section 8 .

\section{Applying to University in the United Kingdom}

In the United Kingdom, applications to undergraduate higher education are handled by the University and Colleges Applications System (UCAS). The organisation provides a single portal through which prospective students are allowed to make up to five applications (for a $£ 26$ fee) to as many undergraduate programmes. Generally, applications are made before high school qualifications are completed, sometime between September and January of the final year of high school (Year 13). Upon reviewing the evidence provided in an application, Universities most commonly either reject applicants or make conditional offers to them. Conditional offers promise to accept an applicant provided they meet a set of criteria in their final high school qualifications. After outcomes are received from Universities, but before high school results are announced, applicants have to select one of their offers as firm (their top choice), and a second offer as insurance (second choice). Once results are published, Universities decide whether to accept each firm applicant based on whether they meet (or closely meet) their offer. Should an applicant be rejected by their firm choice, they may be accepted by their insurance choice. In cases where applicants do not match the offer criteria for either their firm or insurance choice, they are released in clearing. Applicants can also release themselves into clearing should they have changed their mind about attending their firm or insurance institution. Applicants in clearing can then search for available places at any University, but this carries some risk as many Universities might then be at capacity.

In 2019 [1], there were a total of 706,435 applicants, and 541,240 of these were accepted at an HE institution, the 2019 data is used due to 2020 being an exceptional year because of the COVID-19 global pandemic and so the number of applications and acceptances may not be comparable to previous years. Almost $69 \%$ of accepted candidates were accepted into their firm choice and close to $7 \%$ of accepted candidates were accepted into their insurance choice. Nearly $14 \%$ of 
accepted candidates were accepted through clearing, with about a quarter of these applying directly during the clearing period. The only other large acceptance route is the Records of Prior Acceptance (RPA), which accounts for $8 \%$ of all acceptances in 2019 . The remainder, roughly $2 \%$ of acceptances, happen via a variety of routes (UCAS Extra, adjustment or other options).

\section{Classification of subjects}

In order to classify academic subjects, and allow for in-depth data analysis, UCAS and the Higher Education Statistics Agency (HESA) introduced the Joint Academic Coding System (JACS) in 2002. Prior to 2002, UCAS and HESA each had a distinct coding system to serve a similar purpose. JACS is an hierarchical system by design. Academic subjects are split into several subject areas, which are themselves split into principal subjects. These are then split further. JACS codes are made up of a letter followed by three digits. Loosely speaking, the letter represents the subject area, the second digit specifies the principal subject, and the subsequent two digits refine this even further. For example,

$$
\text { F - Physical Sciences }
$$

$$
\text { F3 - Physics }
$$

\section{F34 - Mathematical \& theoretical physics}

F342 - Quantum mechanics

It is not required for academic subjects to be specified below the level of principal subject. For example, physics courses can be assigned the code F300. It is also possible to assign multiple JACS codes to academic subjects and programmes, with a weight. For example, a programme titled Mathematics and Computer Science may be coded as 50\% G100 (Mathematics) and 50\% I100 (Computer Science).

It is important to note that UCAS course codes (the codes used by UCAS to identify each programme at a given HE institution) are often similar to JACS codes, but are not necessarily linked. Institutions try to pick UCAS course codes that are evocative of the JACS codes for the course content, but will necessarily need to have a distinct UCAS code for each of the programmes they offer in a specific area (e.g. BEng, MEng, programmes with a specialisation or intercalated years, etc.). As a result, the JACS code assigned to a specific course can be assigned independently. For example, an undergraduate programme in Theoretical Physics may be identified 
with the F342 UCAS code at one institution, but it may be classified as a simple physics course (F300) or as a Mathematical \& Theoretical Physics course (F340). It is probably unlikely for it to be classified as Quantum Mechanics (F342), as that would likely be too specific for an undergraduate course.

One of the problems with the JACS approach is that its rigid hierarchical nature results in a limit to the number of subjects that can fit within a principal subject or subject area. Indeed, there can only be 9 principal subjects within a subject area, and the principal subjects themselves can only be broken down into 9 subjects. It is therefore easy to run out of space in the coding frame in some instances. For this reason, UCAS and HESA designed a new system, the Higher Education Classification of Subjects (HECoS). This is a flat structure where each subject is assigned a 6 digits code. There is no structure to these codes. Codes were initially randomly generated for the list of subjects, and new subjects are simply given a new code. For example, Physics is 100425 and Chemistry is 100417. As the HECoS codes are new, they are not necessarily linked and mapped to JACS: there may be multiple suitable HECoS codes for a course previously sitting against a JACS code, or there may be a single suitable HECoS code for something that used to be defined by a combination of JACS codes. UCAS and HESA produced mapping documents to help institutions decide how to code their progammes using the HECoS system. The HECoS system was used alongside the JACS system in 2019 and 2020, and is expected to fully replace the JACS system in 2021.

In order to maintain a hierarchical approach to the classification of subjects, UCAS and HESA created the Common Aggregation Hierarchy (CAH). The CAH system categorises $\mathrm{HECOS}$ subjects so that data on related programmes can be aggregated appropriately. Within the CAH system, there are three levels of hierarchy, each level defined by a 2 digit code. This approach does run into the same problem as the hierarchical JACS system, but using 2 digits for each level allows for up to 99 items to be nested under the parent level. For example, using v1.3 of CAH,

$$
\text { CAH07 - Physical Sciences }
$$

CAH07-01 - Physics and Astronomy

$$
\text { CAH07-01-02 - Astronomy }
$$

There may then be multiple HECoS codes mapped to the $\mathrm{CAH}$ level 3 code. CAH v1.3 is expected to come into use in July 2021. 
In addition to being adopted by UCAS and HESA for data reporting purposes, these subject classification systems are also used by other government agencies. For example, the Foreign, Commonwealth and Development Office uses the $\mathrm{CAH} / \mathrm{HECoS}$ system to identify the subject area of programmes for visa purposes in their Academic Technology Approval Scheme (ATAS).

\section{UCAS and HESA data}

UCAS and HESA both publish some of their data free of charge. In the fall of each year, UCAS publishes an end-of-cycle report that includes data for applicants of that year. The latest UCAS data available was published in the fall of 2020 [2], and includes data about applicants that applied between September 2019 and clearing in 2020. HESA publishes data on the student population in January/February of each year, for the prior academic year. The latest HESA data available at the time of writing was published in February 2021 [3], and includes data on students for the 2019-2020 academic year. Both organisations publish data disaggregated by principal subject, rounding numbers to multiples of 5 so that no individual applicant or student can be identified. In the case of HESA some data is aggregated at the level of subject area.

There are some key differences in terms of how UCAS and HESA publish their data, and one of these differences is rather important, affecting how data on combined subjects are reported. This is when a programme of study is linked to more than one JACS or HECoS code, with a weighting to represent the relative importance of each subject in that programme. In the case of UCAS, data on programmes where linked principal subjects are all weighted $50 \%$ or less are all aggregated in a separate category of combined subjects in a way that it would be impossible to separate data for a specific principal subject. Data on programmes where there is a linked principal subject weighted more than 50\% (a major) are reported wholly within that major principal subject.

HESA takes a different approach. For programmes linked to multiple principal subjects, a Full Person Equivalent (FPE) rate is assigned to each principal subject, in accordance with the weightings specified by the institution offering that programme. For example, in an hypothetical world where 100 students apply to study a jointhonours programme in Theoretical Physics and Applied Mathematics (coded as 
$50 \%$ Physics and 50\% Mathematics) and then join that programme, UCAS would not show any data about those applicants under either Physics or Mathematics, whereas HESA would attribute 50 FPE to Physics and 50 FPE to Mathematics. This is particularly important for MSE, as we will see in the next section.

In addition to the openly available data, UCAS and HESA sell a range of data services to Universities and other institution. The analysis in this report is entirely based on the openly available data.

\section{Coding of Materials Science and Engineering}

One of the problems MSE faces as a discipline is highlighted by how it is represented within the JACS, HECoS and CAH. Within JACS, a number of principal subject codes are relevant to MSE. These are

$$
\text { F - Physical Sciences }
$$

F2 - Materials Science

$$
\mathrm{H} \text { - Engineering }
$$

$$
\text { H1 - General Engineering }
$$

H16 - Bioengineering, biomedical engineering \& clinical engineering

$$
\text { H161 - Biomaterials }
$$

$$
\mathrm{J}-\text { Technologies }
$$

$$
\text { J2 - Metallurgy }
$$$$
\text { J3 - Ceramics \& Glass }
$$$$
\text { J4 - Polymers \& Textiles }
$$

J5 - Materials Technology not otherwise specified

The first problem is that no single code captures the essence of MSE. Secondly, the most relevant codes, Materials Science (F2) and Materials Technology (J5), are are in two distinct subject areas. Thirdly, some of these principal subjects include subjects that are generally not relevant for our discipline. For example, J4 (Polymers \& Textiles) includes Machine Knitting (J441) and Commercial Tailoring (J442).

The fact that no single code captures MSE is particularly problematic, as some institutions may be tempted to code their MSE programmes as 50\% Materials Science and 50\% Materials Technology not otherwise specified. UCAS would aggregate data from these programmes to data from all combined programmes of science and engineering/technologies. HESA would do marginally better, and report data using 
FPEs. However this can have dramatic ramifications when thresholds need to be met in order for data to be published. For example, lets assume that there are 40 students on such a combined programme, and that HESA requires a threshold of 30 FPEs in order to publish data, employment statistics or National Student Survey (NSS) output for that programme may not be available.

Similar issues are encountered when looking at the representation of MSE within $\mathrm{CAH}$ and HECoS. The relevant codes are (CAH version 1.3):

CAH10 - Engineering and Technology

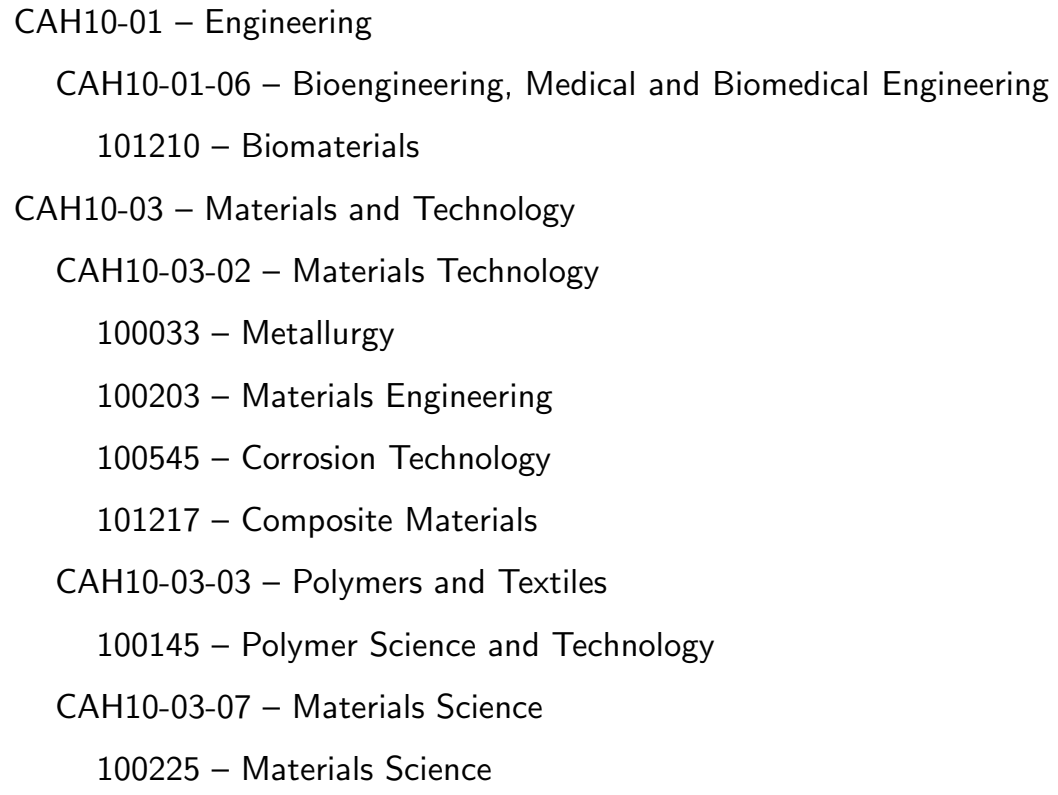

Since CAH version 1.3, which will be implemented in July 2021, MSE-related subjects have been grouped under a single level 1 area (Engineering and Technology). This has been a welcome change relative to $\mathrm{CAH}$ version 1.2 , which placed Materials Science amongst the level 1 Physical Sciences area. The two most relevant HECoS codes (Materials Science and Materials Engineering) are in a single level 2 subject group, Materials and Technology (CAH10-03). However, that level 2 subject group includes many other subjects such as maritime technology and biotechnology. If we drop down to the level 3 groupings, the two most relevant HECoS codes are split in two subject groups, Materials Technology (CAH10-0302) and Materials Science (CAH10-03-07), which leads to the same issues we have encountered when exploring the JACS system. In addition, similar to JACS, relevant level 3 groupings include other subjects that may not be classified as MSE: 
Polymers and Textiles (CAH10-03-03) includes Leather Technology (100210) and

Textiles Technology (100214).

It can be seen that, both within JACS and in HECoS/CAH, Biomaterials appears somewhere else entirely. This could also be a concern. If an institution decides to map an MSE programme with a specialisation in Biomaterials to the Biomaterials subject with a weighting $>50 \%$, data pertaining to that programme will be aggregated with Bioengineering or General Engineering rather than any of the MSE-related subject groups. Even though this is unlikely, and it would be more common for an undergraduate programme in MSE to be mapped to Biomaterials with a weighting of $\leq 50 \%$, there are still issues with meeting reporting thresholds for smaller student populations.

\section{Applications to study Materials Science and Engineering}

Having covered how the undergraduate application process works in the UK in Section 2 and how UCAS and HESA classify subjects in Section 3 we can now start looking at the available data on UCAS applications. Using JACS codes, we can look at data for every single UCAS cycle since 2007, as HECoS/CAH data has only been published in 2019 and 2020. Due to the issues presented in Section 5 we decided to isolate MSE applications and acceptances by summing data for Materials Science (F2) and Materials Technology (J5). We have to ignore both J4, which contains Polymer Technology, and H1, which contains Biomaterials, as both of these principal subjects include many irrelevant detailed subjects. We also opt to ignore Metallurgy (J2) and Ceramics \& Glass (J3). Applications in the Ceramics \& Glass principal subject can be attributed to art programmes, while there have been very few applications in Metallurgy in recent years. With the withdrawal of Metallurgy programmes at the University of Sheffield and the University of Birmingham, we infer from the data that no programmes in the UK are mapped to Metallurgy with a weighting $>50 \%$.

Using this approach, we can see that UCAS received 1815 applications to MSE courses in 2020. Assuming each applicant applies to 4-5 MSE courses (the majority of applicants opt to apply for the same subject at different institutions), we can therefore estimate that at least 400 applicants have applied to MSE courses in 2020. This is an underestimation as the University of Birmingham alone received 


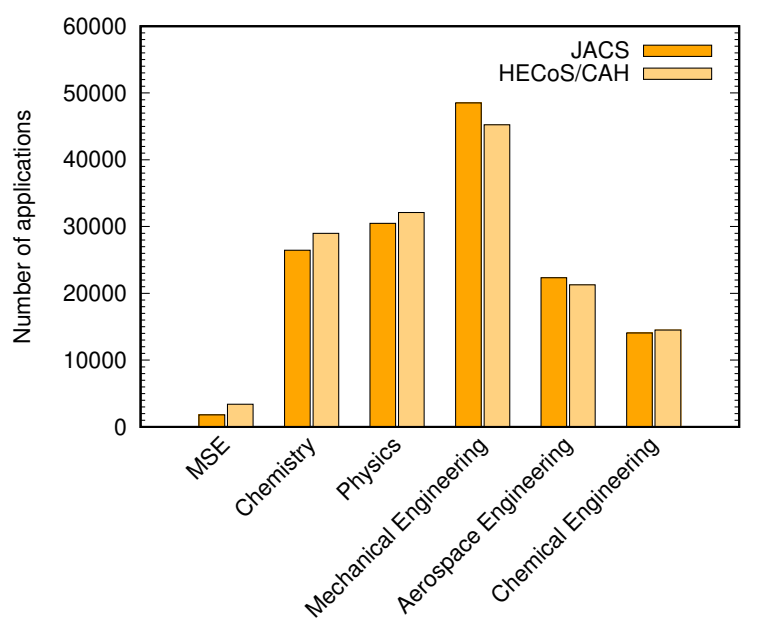

Figure 1: Comparison of 2020 application numbers to MSE and related subjects using JACS and $\mathrm{HECOS} / \mathrm{CAH}$

applications from 300 individual applicants to MSE programmes, and it is one of 10 institutions offering UG MSE programmes. The underestimation is most likely due to two reasons. Firstly, some MSE programmes may either have been miscoded, or may be mapped to a more general subject (e.g. H100, General Engineering). Secondly, some Universities possibly coded their MSE programmes as combined subjects, perhaps mapping 50\% to Materials Technology and 50\% to Metallurgy. With the advent of $\mathrm{HECOS}$ and $\mathrm{CAH}$ codes, the HECoS subjects of Materials Technology and Metallurgy sit within the same $\mathrm{CAH}$ level 3 subject group. Other Universities may have previously mapped MSE programmes to multiple JACS codes but may have opted for simplicity when transitioning to $\mathrm{HECoS}$, perhaps due to the possibility of a subject-level Teaching Excellence Framework (TEF). If we sum applications in Materials Science and Materials Technology CAH level 3 subject groupings, we get 3405 applications in 2020. This number is much closer to an estimation of the number of applications received to Materials Science and Engineering programmes at the 10 institutions offering such programmes.

A comparison of applications numbers as obtained using JACS versus $\mathrm{HECOS} / \mathrm{CAH}$ for various subjects can be seen in Figure 1 . There appears to be always a difference, although that difference is relatively larger in the case of MSE for reasons discussed previously. We note that the number of applications to Chemistry and 

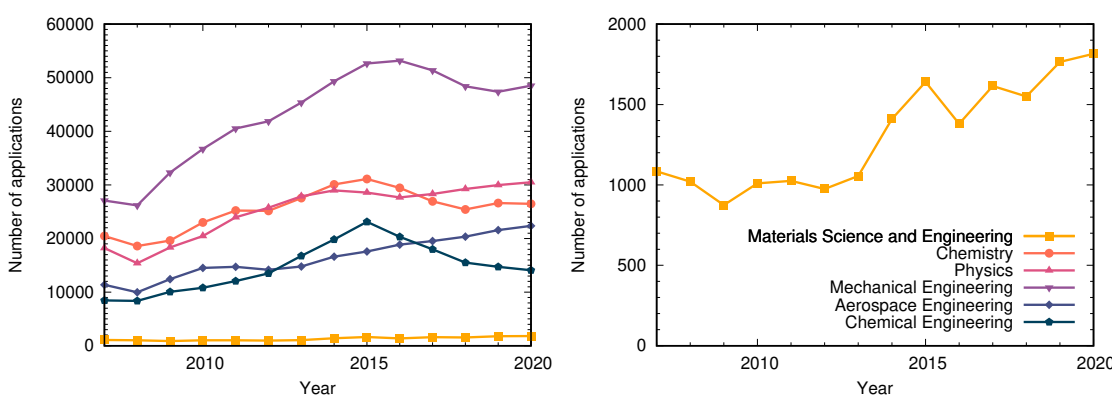

Figure 2: The total number of UCAS applications made to Materials Science and Engineering, Chemistry, Physics, Mechanical Engineering, Aerospace Engineering and Chemical Engineering from 2007 to 2020

Physics also increases when looking at the HECoS/CAH data, while a drop is observed for Mechanical Engineering. Without knowledge of how Universities map their programmes to JACS and HECoS codes, it is impossible to fully establish the reasons for these differences.

Even though the JACS data underestimates our number of applications, we believe it captures a representative sample of MSE applicants. Applicants that apply to MSE programmes that are coded as combined subjects or mapped to something else entirely will also apply to MSE programmes that are mapped against a single principal subject. The proportion of applications from women, overseas applicants, and other applicant characteristics are consistent whether one looks at JACS or HECoS data. It is probably more robust to use HECoS data, as larger application numbers mean it is easier to meet reporting thresholds when data is disaggregated into several categories (e.g. ethnic background). On the other hand, JACS data provides a precious historical perspective as the data stretches back to 2007 .

The total number of applications to MSE compared to applications to Chemistry, Physics, Mechanical Engineering, Aerospace Engineering and Chemical Engineering is shown in Figure 2. Roughly speaking, the number of applications to MSE are an order of magnitude lower than other disciplines we compare to. We have observed significant growth in application numbers, but this is matched to the growth observed in applications to Mechanical Engineering, Aerospace Engineering, and Physics. Chemical Engineering and Chemistry appear to have had sustained growth in the number of applications up until 2015, followed by a significant drop in the 


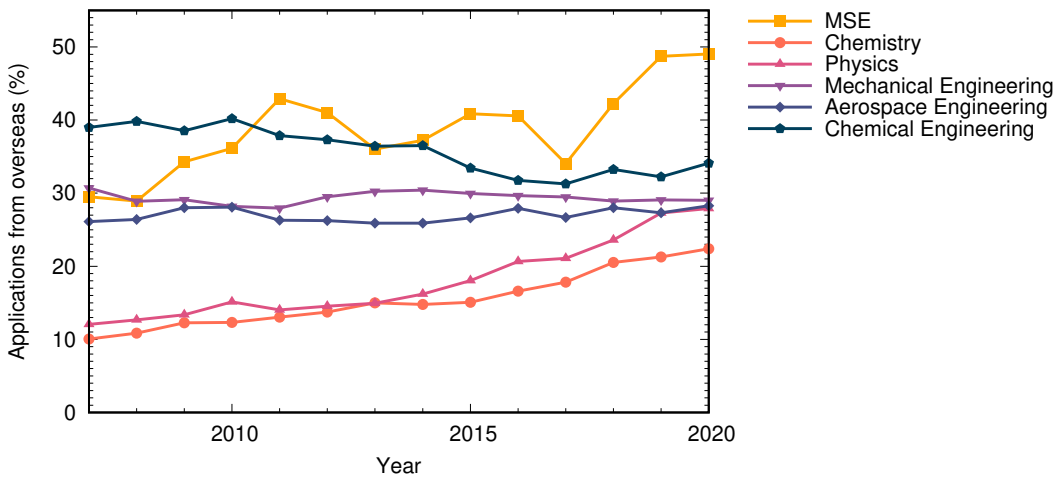

Figure 3: The percentage of applications from overseas applicants made to Materials Science and Engineering, Chemistry, Physics, Mechanical Engineering, Aerospace Engineering and Chemical Engineering from 2007 to 2020

last 5 years.

We would not expect MSE to be as popular as Mechanical Engineering, and it is natural to ask ourselves what an appropriate number of applications should be. To do this, we can look at the proportion of applications that are made from overseas applicants, which we define as anyone not domiciled in the UK. This is shown in the left panel of Figure 3 It can be seen that applications to MSE from overseas applicants have increased significantly, from 30\% to 50\%. At 50\%, the rate of applications from overseas applicants is considerably higher than all other disciplines we compare our data to. Chemical Engineering, the second most popular discipline, received $35 \%$ of its applications for overseas, and it appears this proportion has steadily declined since 2007. Applications from overseas to other engineering disciplines have largely remained unchanged, while Physics and Chemistry have observed significant increases, but the proportion of applications from overseas applicants remains low.

Two things could explain the relatively high proportion of applications from overseas applicants: either MSE is relatively more popular abroad than in the UK, or the UK is seen as a destination country where to study MSE. 


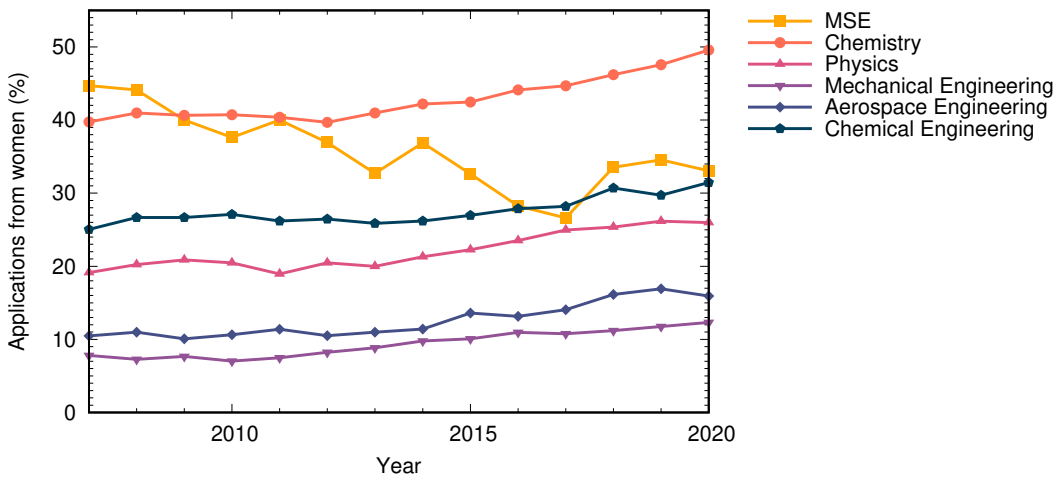

Figure 4: The percentage of applications from women applicants made to Materials Science and Engineering, Chemistry, Physics, Mechanical Engineering, Aerospace Engineering and Chemical Engineering from 2007 to 2020

\section{Characteristics of applicants pool}

It is useful to study the characteristics of the applicants pool, in order to determine how accessible the discipline is to the wider public. Anecdotal evidence suggests that many MSE students stumble into the subject almost by mistake, while exploring opportunities in science and engineering through friends and family working in high-skilled jobs. Others are aware of the subject because a family member or a family friend has studied it or works in the sector. University tutors in schools are generally less aware of it, and may be inclined to direct their students to better-known disciplines such as Mechanical Engineering, Physics or Chemistry.

\subsection{Women}

UCAS publishes the data split by the stated gender of the applicant, which UCAS treats as a binary variable at this stage [4]. We can therefore use this data to study the proportion of applications coming from women, and compare this to similar disciplines. Using JACS, we can look at data from 2007 to 2020, and the fact that applications are divided in two relatively large groups (men and women) means we do not have to worry about issues caused by the data being rounded to a multiple of five.

The proportion of applications from women made to MSE, Chemistry, Physics, Mechanical Engineering, Aerospace Engineering and Chemical Engineering is shown in Figure 4. Although MSE does seem to attract a large proportion of applications 
from women relative to other engineering disciplines (e.g. $12 \%$ for Mechanical Engineering versus $32 \%$ for MSE in 2020), the historical data suggests some soul searching is required. All disciplines that we have looked at show a steady increase in the proportion of applications coming from women in the 13 years to 2020 . The only exception seems to be MSE, where the proportion of applications from women has dropped from $45 \%$ to $33 \%$ in the last 13 years. This is surprising and it implies efforts to reduce the gender gap are either insufficient or ineffective in MSE, while progress seems to have been made in other disciplines. The current proportion of applications coming from women is confirmed when looking at the HECoS/CAH data, where we find $36 \%$ of applications to MSE came from women in 2020. This suggests it is unlikely that the trend is a result of biases introduced by the subject coding system.

Observation of the raw numbers reveals that while applications from women have only grown by $25 \%$ since 2007 , applications from men have more than doubled. The reason for the relative growth in popularity of MSE amongst men is unclear. Qualifications required to study the subject across the sector have not changed considerably, and these are similar to other sciences and engineering disciplines. The uptake of A-level subjects like physics and chemistry from girls relative to boys has increased in recent years, while it has remained largely unchanged for mathematics. We can only infer that the work done by the MSE community to promote the discipline over the last two decades has been more successful at convincing men to apply for the subject.

We would ideally like to be able to confirm whether this downward trend is observable when looking at the proportion of women registered for MSE courses as reported by HESA. Unfortunately, to the best knowledge of the authors, HESA openly reports the number of registered students split by personal characteristics at a higher level, by JACS subject area or CAH level 1 category. This would not give us sufficient granularity to isolate MSE programmes.

\subsection{Ethnic background}

We can now look at the number of applications split by ethnic group. UCAS reports data split in the following categories: asian, black, mixed, other, white, and unknown (prefer not to say). Due to the higher number of groups, and the fact that some of these groups represent a small proportion of the applicants, we are 


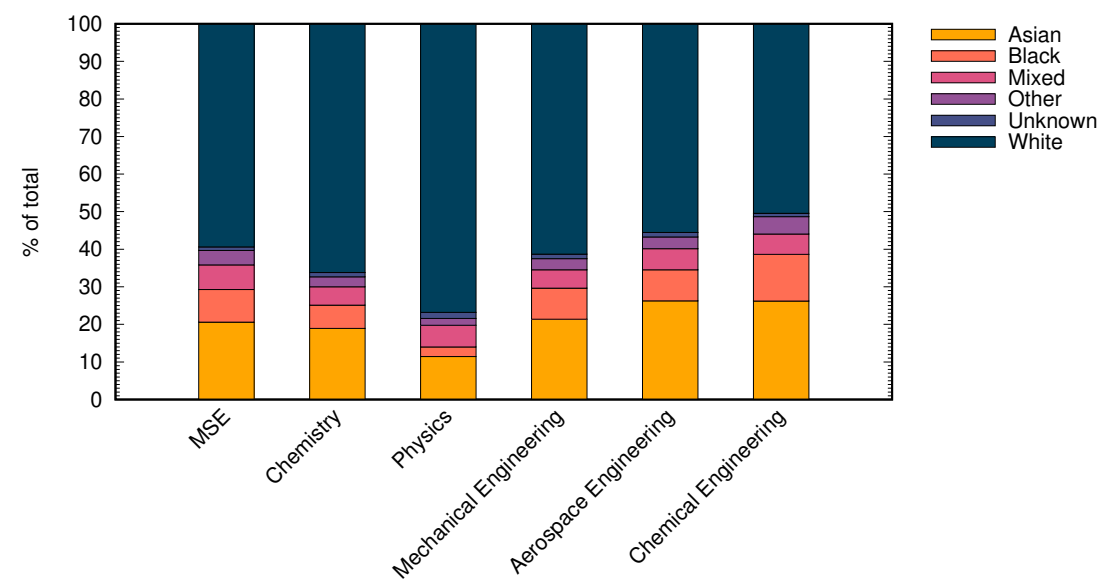

Figure 5: The percentage of home applications from people of asian, black, mixed, other, unknown and white background made to Materials Science and Engineering, Chemistry, Physics, Mechanical Engineering, Aerospace Engineering and Chemical Engineering in 2020

restricted to use the $\mathrm{HECoS} / \mathrm{CAH}$ data, and lose the historical perspective. There are simply not enough applications in the JACS data to meet the reporting threshold for all groups dating back to 2007.

The percentage of total applications to MSE and comparator disciplines from the UCAS ethnic groups in 2020 is shown in Figure 5. We note this only includes home applicants, and excludes applications from overseas and the European Union. MSE appears to sit somewhere in between science and engineering disciplines. Engineering disciplines seem more successful at attracting applicants from asian, black and mixed backgrounds compared to MSE.

\subsection{Socioeconomic background}

UCAS data also reports on the applicants' socioeconomic background. In the UK, there are several methods to measure this. UCAS publishes data using two distinct methods. One is the Participation Of Local ARea (POLAR) classification, which classifies residential areas (i.e. postcodes) based on the proportion of young people progressing to and participating in higher education. The POLAR system splits UK postcodes into 5 groups, or quintiles. Postcodes in quintile 1 have the lowest rate of participation while postcodes in quintile 5 have the highest rate of participation. The other measure used by UCAS is the Index of Multiple Deprivation 


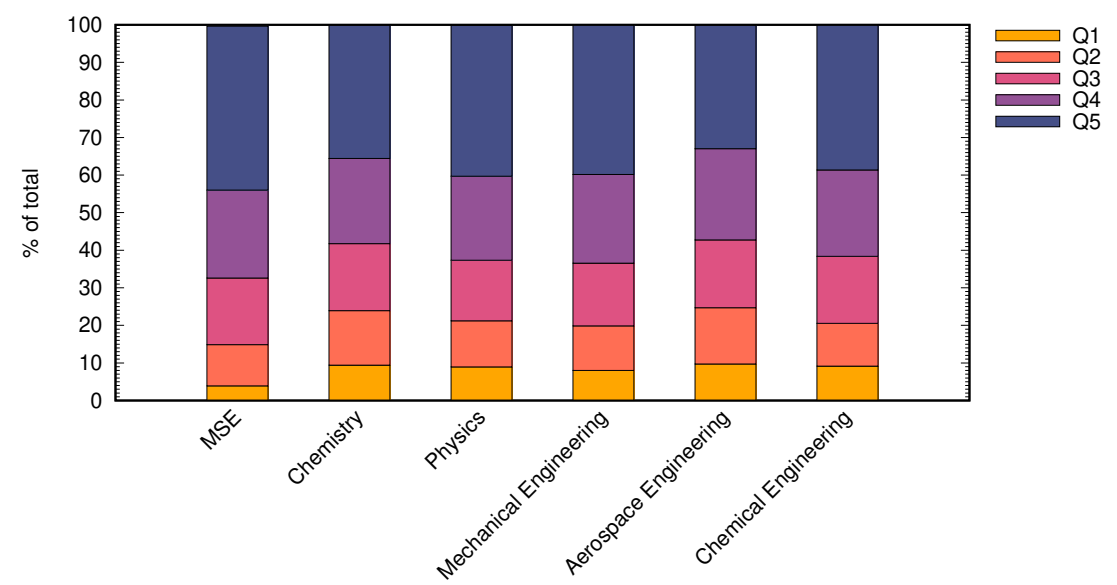

Figure 6: The percentage of home applications classified by the POLAR4 category of the applicant made to Materials Science and Engineering, Chemistry, Physics, Mechanical Engineering, Aerospace Engineering and Chemical Engineering in 2020

(IMD). This approach also classifies UK postcodes into 5 quintiles, with quintile 1 being the most deprived and quintile 5 being the least deprived. The index combines data on income, employment, education, health, crime and housing to rank all postcodes in terms of deprivation. It is important to note that the social makeup within a given postcode may be diverse. As expected, the position of postcodes within these ranking tables may change, so new versions of these classifications are published periodically. For example, the most recent version of POLAR is version 4 (POLAR4).

We first can look at applications split by the POLAR4 classification of the applicant, as shown in Figure 6. Compared to other established science and engineering disciplines, we appear to have a low proportion of applications coming from postcodes with lower likelihood of participation in higher education. Close to $70 \%$ of applications to MSE programmes come from postcodes with a higher than average participation in higher education.

This is not surprising: the disciplines we compare to are well known amongst the public. Young people from low POLAR quintile postcodes will more likely know of others studying those disciplines, and may be completely unaware of MSE. Teachers and university advisors at their schools may focus on promoting the study of better known disciplines. In addition, MSE programmes are less widely available than more 
established science and engineering disciplines, and may therefore be less accessible to those less able to relocate to attend university.

\section{Conversion to students}

Within HE institutions, it is fairly common to monitor the conversion rates at key stages in the admissions cycle. For the purpose of our study, three conversion rates are particularly important:

- Offers to applications ratio - this is the number of conditional and unconditional offers made to study a subject relative to the number of applications made to that subject

- Acceptances to offers ratio - this is the number of acceptances in a particular subject relative to offers made to study that subject

- Registered students to acceptances ratio - this is the total number of year 1 registered students in a particular subject relative to the number of acceptances in that subject

It is worth pointing out that these conversion rates do not really tell us anything about the experience of an individual applicant. Since published data is anonymous, it is impossible to know, for example, how many offers an individual applicant has received, or how clustered these offers are within the applicant pool. Also, students might apply in one academic year, and defer their entry to the following academic year, and there are a number of students that apply directly through clearing without having made main scheme applications. The simplest thing we can do is to take the ratio of the total numbers of applications, offers, acceptances and registered students, and be careful not to attach additional unsupported meaning to these figures.

The calculated conversion rates are shown in Figure 7. The data for the offers to applications ratio and acceptances to offers ratio spans from 2007 to 2020 (available through UCAS). For the ratio of registered students to acceptances, we have to use HESA data, and this limits our time window. More recent HESA data is available, but this is published using $\mathrm{HECoS} / \mathrm{CAH}$, and we cannot directly compare to the JACS data used for all other years. 

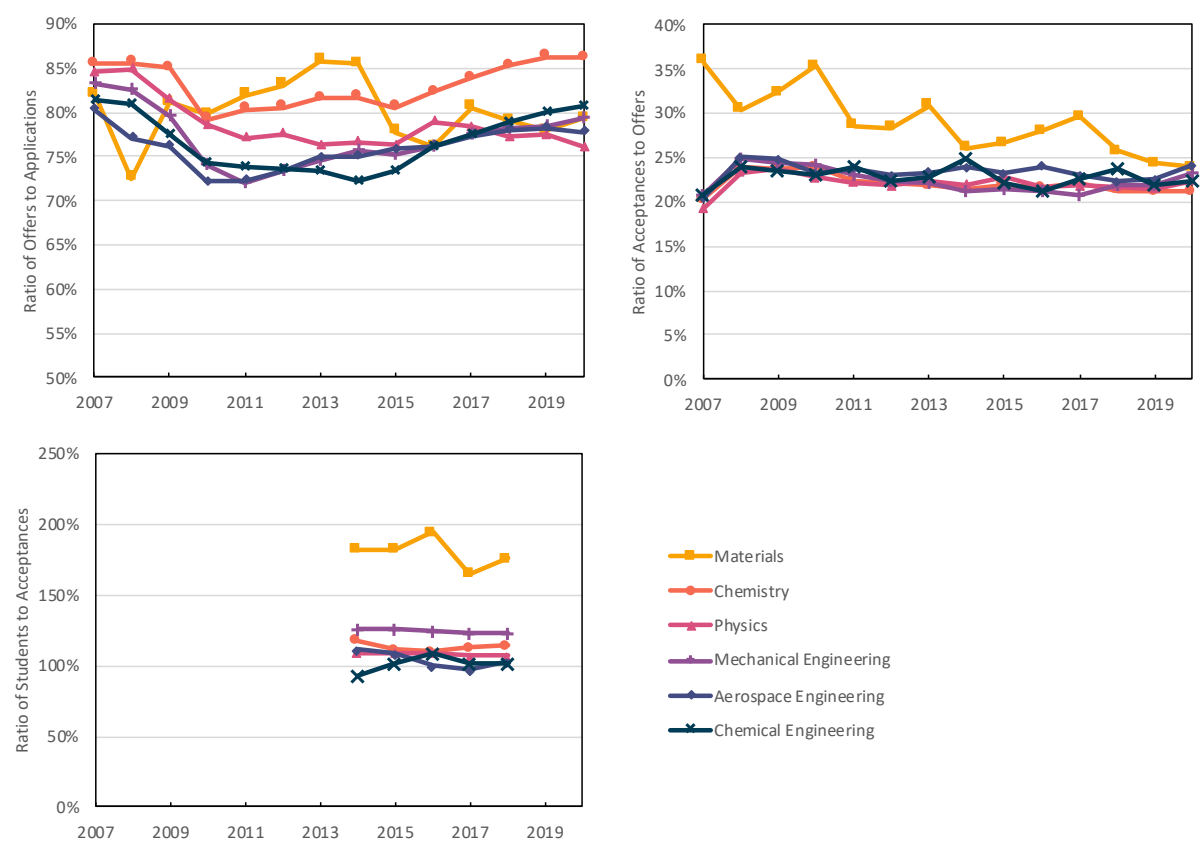

Figure 7: The offers to applicants ratio (top left), acceptances to offers ratio (top right) from 2007 to 2020, and registered students to acceptances ratio (bottom) from 2014 to 2018 for Materials Science and Engineering, Chemistry, Physics, Mechanical Engineering, Aerospace Engineering and Chemical Engineering 
It can be seen that the ratio of the number of offers to the number of applications generally fluctuates between $70 \%$ and just over $85 \%$. Differences between subjects are not significant. The situation is different for the other two conversion ratios.

When looking at the ratio of acceptances to offers, MSE appears to behave differently compared to other subjects. The ratio appears higher, on average, for MSE relative to other disciplines. This either suggests MSE offer holders are more likely to satisfy the acceptance criteria for their offers relative to other disciplines, or that Universities are more flexible when making decisions on students that are narrowly missing their conditional offers. The difference between MSE and other disciplines appears to be reducing overtime, and reasons for this are unclear from the data.

The difference between MSE and other disciplines is even more obvious when looking at the ratio of the number of students to acceptances. It is important to remember that the numerator here is obtained from HESA, which includes FPE fractions for combined programmes, while UCAS does not do this (see Section 4). It is therefore expected that these ratios might be above $100 \%$ : there will be additional fractions of students that are registered on combined programmes. MSE here appears to be a clear outlier, with the number of registered students appearing to be almost twice the number of accepted students.

This is partly due to the differences between how HESA and UCAS report their data, but it is unlikely this is the only explanation. We can investigate this by looking at the number of registered MSE year 1 students reported by HESA. According to HESA there were 555 students (FPE) registered under F2 and J5 JACS codes in the UK in the academic year 2018/2019. This is much higher than the 315 acceptances in those same JACS codes in the UCAS cycle leading to that academic year. The majority of the 555 registered year 1 students is in J5 (410) and the rest are in F2 (145). We would ideally like to identify the proportion of 'whole' students vs the number of FPE that is a result of fractions coming from combined programmes. This is unfortunately impossible to determine from the freely available data.

In order to estimate how many of these students are FPE fractions, we can look at which institutions these students are registered in to study MSE. HESA publishes this, but as a total of UG students (FPEs) so that it is not possible to determine the number of first year students registered under a particular JACS 
code at a specific institution. Nevertheless, the institution data may shed some light. In the academic year 2018/2019, a total of 1985 FPE UG students (all years) was registered on programmes mapped to F2 (520) or J5 (1465) across the UK. The first obvious thing to check is that no institution has students in both, which implies a combined programme mapped to both F2 and J5. This is indeed not the case, as all institutions either report registered students in F2 or report registered students in J5. The only exception is the University of Birmingham, reporting a very small number of students (5) in F2 due to a programme now withdrawn, and the University of Derby, reporting small numbers in both JACS codes (10 and 15 respectively).

Next, we can look at how these students are distributed. Only 9 institutions report 100 or more students in the relevant JACS codes. These are all institutions that have single-honours programmes in MSE that are probably wholly mapped against a single JACS code. These institutions may also offer major/minor combinations, but these would not be classified as combined subjects according to UCAS. The total number of FPE students registered at these 9 institutions is 1855 . Unless one of these 9 institutions offers a combined programme with another discipline that is taken by a significant number of students, we can conclude that at least over $90 \%$ of FPE students registered for either F2 or J5 JACS codes are registered for programmes that are wholly mapped against one of those two JACS codes, or have a major/minor configuration where one of those two JACS code carries a weight higher than $50 \%$.

Assuming this remains roughly constant, we can infer that roughly 500 of the year 1 students (FPE) registered to study on programmes mapped to either F2 or $\mathrm{J} 5$ is actually registered on programmes that are not considered combined according to UCAS. This would lower the students to acceptances conversion rate from $176 \%$ to $159 \%$. We therefore conclude that differences in how UCAS and HESA report their numbers is not the cause for MSE behaving as an outlier.

Instead, we suggest that a significant proportion of the registered MSE students in year 1 is a result of internal transfer processes after the UCAS cycle is complete. 
456 [1] UCAS, 2019 End of Cycle Report.

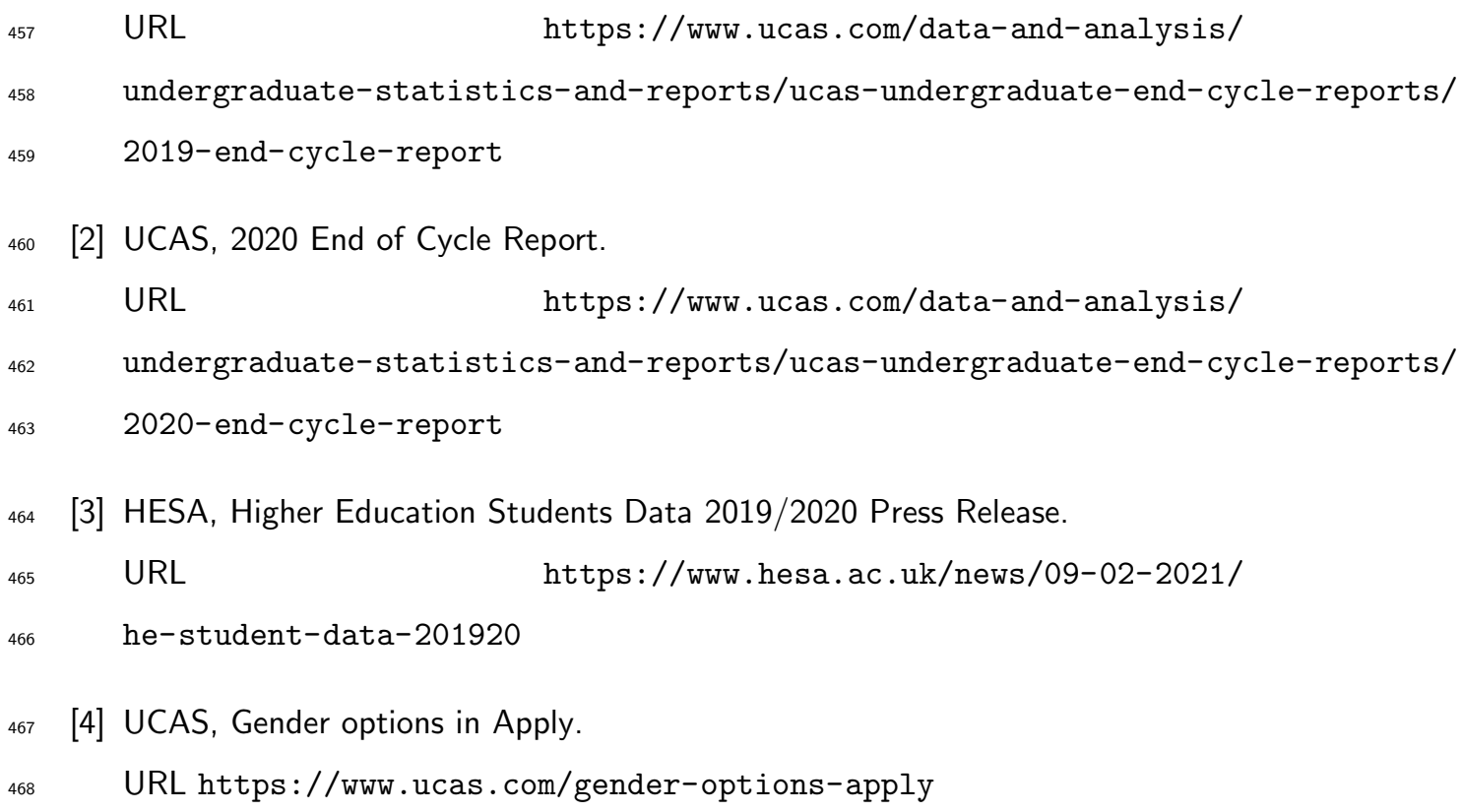

